\title{
rf acceleration with harmonic number jump
}

\author{
Alessandro G. Ruggiero \\ Brookhaven National Laboratory, P.O. Box 5000, Upton, New York, USA
}

(Received 6 September 2006; published 17 October 2006)

\begin{abstract}
We have recently considered acceleration of protons and heavy ions in a fixed-field alternating-gradient accelerator with nonscaling lattice and linear field profile. To avoid the problem of frequency modulation for acceleration of low-energy beams over a too short period of time, and to boost the acceleration rate, the method of harmonic number jump (HNJ) was proposed. This method allows the use of constant frequency acceleration, for instance using superconducting cavities, despite the fact that the beam velocity may vary considerably. Of course in this case the accelerating voltage and $\mathrm{rf}$ phase will need to be programmed accordingly as we shall show. We shall study first the motion of synchronous particles, and then of those with deviating initial conditions. We estimate the area and height of the rf buckets that are to contain the beam bunches with the added condition of the HNJ. We finally investigate methods to allow the program of energy gain as required by the HNJ method, including the effect of the cavity transit time factor.
\end{abstract}

DOI: 10.1103/PhysRevSTAB.9.100101

PACS numbers: $29.20 .-\mathrm{c}$

\section{ACCELERATION OF SYNCHRONOUS PARTICLES}

Let us assume that the fixed-field alternating-gradient (FFAG) ring with nonscaling lattice and linear field profile [1] is made of a number of rf cavities equally spaced, and that the lattice properties and structure between two consecutive cavities repeat identically around the ring (see Fig. 1). The acceleration cycle is then thought as a sequence of pair events: an energy kick at the location of the $n$th cavity, followed by an $\operatorname{arc} A_{n}$ that takes the beam from that cavity to the next. We shall investigate the proposed [2] method of acceleration based on the harmonic number jump (HNJ).

Let us start considering a beam made of pointlike bunches; that is, all particles have the same energy, and cross the cavities all together at the same instant. Equivalently each pointlike bunch can be thought to be a synchronous or a reference particle around which all other particles oscillate. The total energy of the reference particle in the $n$th $\operatorname{arc} A_{n}$ is $\mathbf{E}_{n}$, and the period of time that it takes to travel the $\operatorname{arc} A_{n}$ is $\mathbf{T}_{n}=h_{n} T_{\text {rf }}$, where $T_{\text {rf }}$ is the constant rf period, and $h_{n}$ is the rf harmonic number local to the $\operatorname{arc} A_{n}$. Here and in the following, bold face parameters apply to the reference particle. The same parameters in plain face apply to the other particles.

Let $Q$ and $A$ denote, respectively, the charge state and the mass number of the ion particle. The energy gain when crossing the $n$th cavity is

$$
\Delta \mathbf{E}_{n}=\left(Q e V_{n} / A\right) \sin \left(\omega_{\mathrm{rf}} \mathbf{t}_{n}\right)=\left(Q e V_{n} / A\right) \sin \left(\boldsymbol{\phi}_{n}\right),
$$

where $V_{n}$ is the peak voltage, $\omega_{\text {rf }} / 2 \pi$ the rf frequency, $\mathbf{t}_{n}$ the instant of traversal of the cavity, and $\boldsymbol{\phi}_{n}=\omega_{\mathrm{rf}} \mathbf{t}_{n}$ the rf synchronous phase. Both $V_{n}$ and $\boldsymbol{\phi}_{n}$ can vary from cavity to cavity, and, in the same cavity, from turn to turn.

The HNJ method requires that the energy gain is adjusted to cause a change in the travel period $\mathbf{T}_{n}$ in the following $\operatorname{arc} A_{n}$, so that the reference particle is pushed forward or back exactly by $\Delta h \mathrm{rf}$ harmonics and appears in an exactly identical bucket ahead or trailing by $\Delta h \mathrm{rf}$ wavelengths (see Fig. 2). Thus, by denoting

$$
\begin{aligned}
\mathbf{T}_{n} & =h_{n} T_{\mathrm{rf}} \\
\mathbf{T}_{n-1} & =h_{n-1} T_{\mathrm{rf}} \\
h_{n}-h_{n-1} & =-\Delta h,
\end{aligned}
$$

this is accomplished by requiring the boot-strap condition:

$$
\Delta \mathbf{E}_{n}=\beta_{n}{ }^{2} \gamma_{n}{ }^{3} E_{0} \Delta h / h_{n}\left(1-\alpha_{p n} \gamma_{n}{ }^{2}\right) \text {, }
$$

where $E_{0}$ is the ion particle rest energy, $\beta_{n}$ and $\gamma_{n}$ the usual relativistic velocity and energy factors, and $\alpha_{p n}$ the momentum compaction factor local to the $n$th $\operatorname{arc} A_{n}$. The HNJ is executed by combining Eqs. (1)-(3).

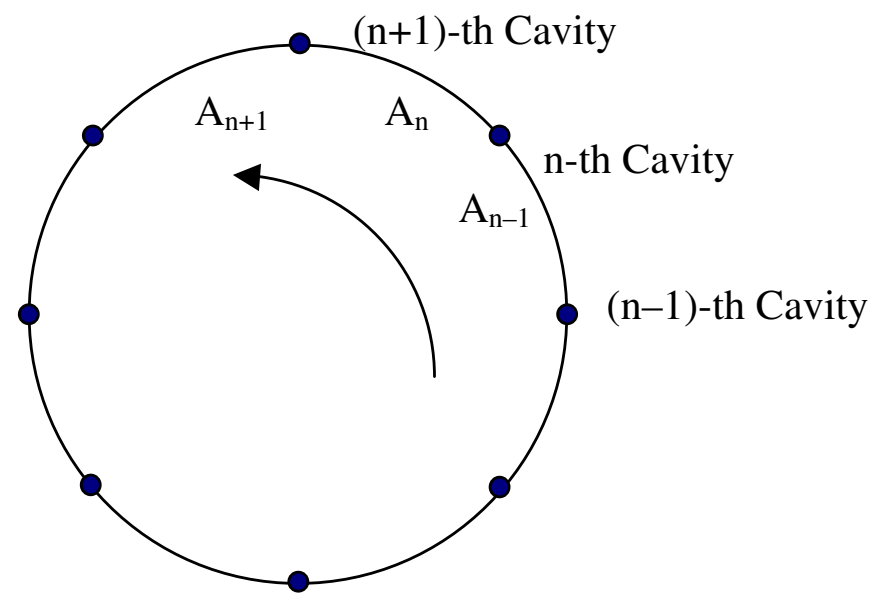

FIG. 1. (Color) FFAG ring with multiple equally spaced rf cavities. 


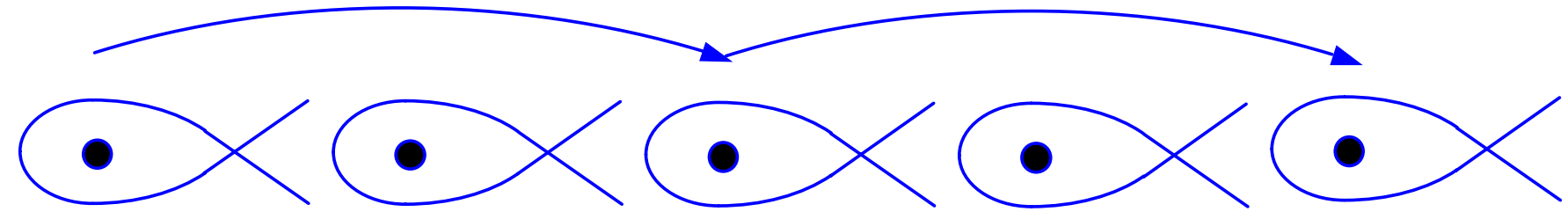

FIG. 2. (Color) rf bucket jump at cavity crossings by $\Delta h \mathrm{rf}$ wavelengths (harmonic number).

\section{ACCELERATION OF NONSYNCHRONOUS PARTICLES}

Let us consider now an off-momentum particle following (or heading) the reference particle with a time difference $\tau_{n}$. It will cross the $n$th cavity at the instant $t_{n}=\mathbf{t}_{n}+\tau_{n}$, and the corresponding energy gain is

$$
\Delta E_{n}=\left(Q e V_{n} / A\right) \sin \left(\omega_{\mathrm{rf}} t_{n}\right)
$$

Subtracting side by side Eq. (1) from Eq. (4) gives the change $\Delta \varepsilon_{n}$ of the energy difference $\varepsilon_{n}=E_{n}-\mathbf{E}_{n}$ after crossing the $n$th cavity

$$
\Delta \varepsilon_{n}=\left(Q e V_{n} / A\right)\left[\sin \left(\boldsymbol{\phi}_{n}+\omega_{\mathrm{rf}} \tau_{n}\right)-\sin \left(\boldsymbol{\phi}_{n}\right)\right] .
$$

In the limit of small delay $\tau_{n}$

$$
\Delta \varepsilon_{n}=\left(Q e V_{n} / A\right)\left(\cos \phi_{n}\right) \omega_{\mathrm{rf}} t_{n} .
$$

The change $\Delta \tau_{n}$ of the time difference $\tau_{n}$, in linear approximation, is given by

$$
\begin{aligned}
\Delta \tau_{n} & =\tau_{n}-\tau_{n-1}=\left(t_{n}-\mathbf{t}_{n}\right)-\left(t_{n-1}-\mathbf{t}_{n-1}\right) \\
& =T_{n}-\mathbf{T}_{n}=-\left(1-\alpha_{p n} \gamma_{n}{ }^{2}\right) \mathbf{T}_{n} \varepsilon_{n} / \beta_{n}{ }^{2} \gamma_{n}{ }^{3} E_{0} .
\end{aligned}
$$

Combining Eqs. (6) and (7) gives the phase (or energy) oscillation equation in the limit of small amplitude,

$$
\Delta^{2} \tau_{n} / \Delta n^{2}+\Omega_{n}{ }^{2} \tau_{n}=0,
$$

where the oscillation angular frequency [using Eqs. (1), (3), (6), and (7)]

$$
\Omega_{n}^{2}=2 \pi \Delta h / \tan \phi_{n} .
$$

\section{MOTION WITHIN rf BUCKETS}

The two canonical equations (5) and (7) can be derived from the following Hamiltonian:

$$
\begin{aligned}
H= & \left(Q e V_{n} / A \omega_{\mathrm{rf}}\right)\left[\cos \left(\boldsymbol{\phi}_{n}+w_{\mathrm{rf}} \tau_{n}\right)+\omega_{\mathrm{rf}} \tau_{n} \sin \left(\boldsymbol{\phi}_{n}\right)\right] \\
& +-\left(1-\alpha_{p n} \gamma_{n}{ }^{2}\right) \mathbf{T}_{n} \varepsilon_{n}{ }^{2} /\left(2 \beta_{n}{ }^{2} \gamma_{n}{ }^{3} E_{0}\right) .
\end{aligned}
$$

The motion of all particles is to occur in rf buckets. The simple analogues for the usual equations for bucket fixed points, and height, and area $[3,4]$ may be obtained from the Hamiltonian of Eq. (10). There is though the exception that the energy gain per cavity is programmed according to the combination of Eqs. (3) and (4). The rate of acceleration cannot be given a priori, but is determined by the required energy gain program. This is most advantageous indeed in
FFAG accelerators where the guiding field does not change with time.

\section{CONSEQUENCES OF THE HARMONIC NUMBER JUMP}

The procedure we have followed applies correctly to the case when beam energy is constantly below the transition energy, namely, when $\alpha_{p n} \gamma_{n}{ }^{2}<1$. We have indeed set Eqs. (1) and (3) explicitly for this case, that requires a decreasing harmonic number as acceleration proceeds. In the opposite case when the beam energy is above the transition energy, namely, that $\alpha_{p n} \gamma_{n}{ }^{2}>1$, the harmonic number will increase monotonically. Thus Eq. (2c) is to be replaced with $h_{n}-h_{n-1}=+\Delta h$, and a minus sign is to be placed in front of the right-hand side of Eq. (3). Obviously, to avoid unnecessary beam losses, the number of bunches, which is a constant during acceleration, ought to be always less than the harmonic number at all times. On the other end, because of the change of the revolution period due to the beam velocity variation, the number of $\mathrm{rf}$ buckets will vary. The actual distribution of the beam bunches with respect to the available buckets during acceleration is shown in Fig. 3(a) for the case of acceleration below the transition energy, and in Fig. 3(b) for the case above.

It is seen that in the case below transition energy the beam longitudinal extension at injection ought to be shorter than the revolution period and at most equal to the revolution period at extraction. That is, the number of

(a)

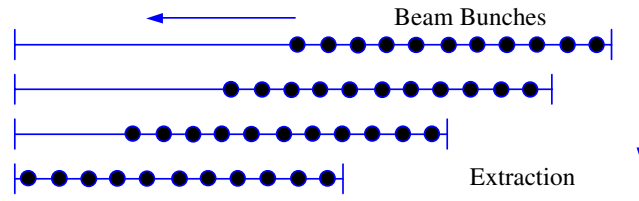

Injection

(b)
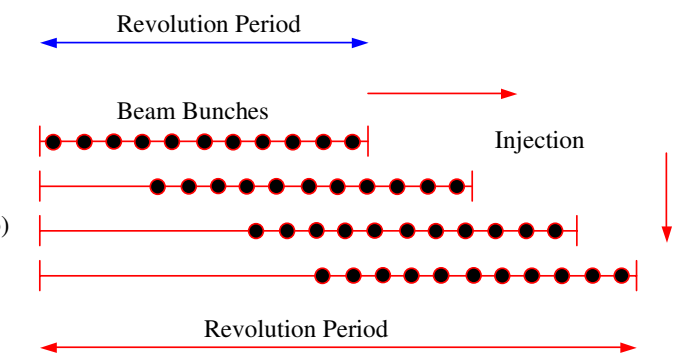

Extraction

FIG. 3. (Color) Bunch to bucket configuration below transition energy (a) and above transition energy (b). 
injected bunches cannot be larger than the rf harmonic number at extraction. The situation is different when the beam is injected at one energy above the transition energy value. In this case the revolution period decreases and the harmonic number increases during acceleration. The ratio of the harmonic number at extraction to that at injection is equal to the ratio of the corresponding values of the beam velocity $\beta c$ for the case above transition energy, and to the inverse of that for the case of acceleration below the transition energy. The creation of a beam gap during acceleration above the transition energy may be useful for a variety of applications, for instance to allow time for the extraction kickers.

\section{ENERGY GAIN PROGRAMMING}

The acceleration method by HNJ requires that the energy gain at the $n$th cavity is given by the combination of Eqs. (1) and (3), that is,

$$
e V_{n} \sin \left(\boldsymbol{\phi}_{n}\right)=A \beta_{n}{ }^{2} \gamma_{n}{ }^{3} E_{0} \Delta h / Q h_{n}\left(1-\alpha_{p n} \gamma_{n}{ }^{2}\right)
$$

As an example, we show in Fig. 4 the energy gain per cavity required for the case of the injector FFAG ring proposed recently for the proton driver of the neutrino factory [5]. What is shown is $e V_{n} \sin \left(\phi_{n}\right)$ versus the actual radial position of the beam during acceleration as directly given by Eq. (11) for $\Delta h=1$. The ring accelerates protons from $400 \mathrm{MeV}$ to $1.5 \mathrm{GeV}$, and has a circumference of $807.091 \mathrm{~m}$. Acceleration occurs at the constant rf frequency of $805.2 \mathrm{MHz}$ with 2 diametrically opposite groups each of 8 equally spaced, independently phased, superconducting, single-gap cavities. Let $\xi_{n}$ denote the average axial field in the cavity made of one single elliptically shaped cell with gap $g$ operating in half-wavelength mode. Let also $\lambda$ denote the rf wavelength. The cavity voltage can then be expressed as

$$
V_{n}=g \xi_{n} \operatorname{TTF}\left(\beta_{0} / \beta\right)
$$

where

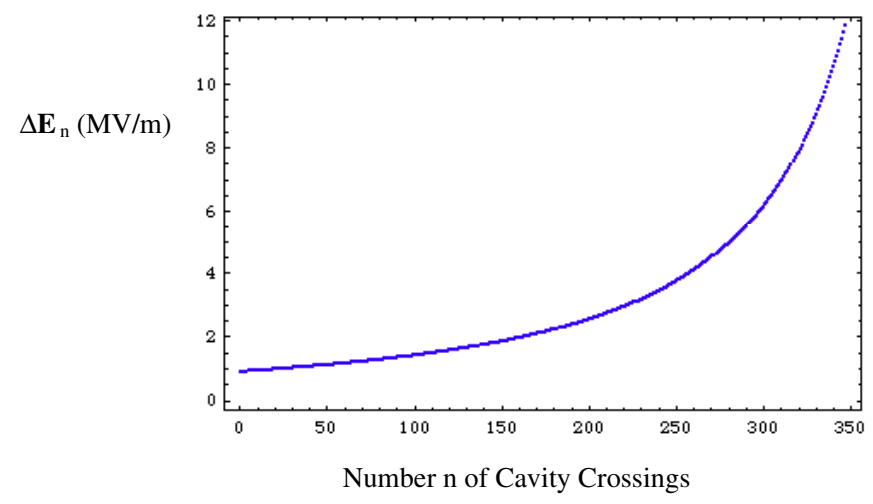

FIG. 4. (Color) Energy gain $\Delta \mathbf{E}_{n}=e g \xi_{n} \operatorname{TTF}\left(\beta_{0} / \beta_{n}\right) \sin \left(\phi_{n}\right)$ vs number $n$ of cavity crossings.

$$
g=\lambda \beta_{0} / 2
$$

and

$$
\operatorname{TTF}\left(\beta_{0} / \beta\right)=\sin \left(\pi \beta_{0} / 2 \beta\right) /\left(\pi \beta_{0} / 2 \beta\right)
$$

is the transit time factor (TTF) that is a function of the beam velocity $\beta$, and $\beta_{0}$ is a reference value that corresponds to the gap $g$ for which the TTF has been optimized. Typically $\beta_{0}$ is adjusted to the value of the beam velocity in the middle of the acceleration cycle [6,7].

The profile of Fig. 4 can be realized with four different modes of operation.

1. Constant rf phase.-The average axial accelerating field $\xi_{n}$ is shown in Fig. 5 across the radial aperture for $\phi_{n}=60^{\circ}$. The realization of such a field profile across the radial aperture is problematic but not impossible. It could be made with ordinary pill-box cavities resonating in TM010 mode but displaced horizontally [2]. A cavity that provides a longitudinal kick proportional to the radial displacement of the beam is the one operating in TM110 mode. Such cavity introduces also transverse deflecting modes that should be evaluated first and their impact to the beam compensated or at least reduced.

2. Constant average axial field $\xi_{n}$.-This is obtained by programming the rf phase during the acceleration cycle. The result is shown in Fig. 6 that corresponds to the same axial field in all cavities $\xi_{n}=15.74 \mathrm{MV} / \mathrm{m}$. That includes also the transit time factor TTF. This mode of operation requires that the rf cavities are properly phased with respect to each other, and that the phase in all cavities varies from turn to turn. In both cases the axial field may be exceedingly too large. It could be lowered with multicell cavities, though at the same time the transit time factor behavior may get more irregular due to the large beam velocity change. It is better to operate with a group of localized single-gap cavities independently tuned from each other as done in this example.

Notice that the largest phase change per cavity group crossing does not exceed $1^{\circ}$, and that it occurs at the end of the acceleration cycle. It may be possible to combine this

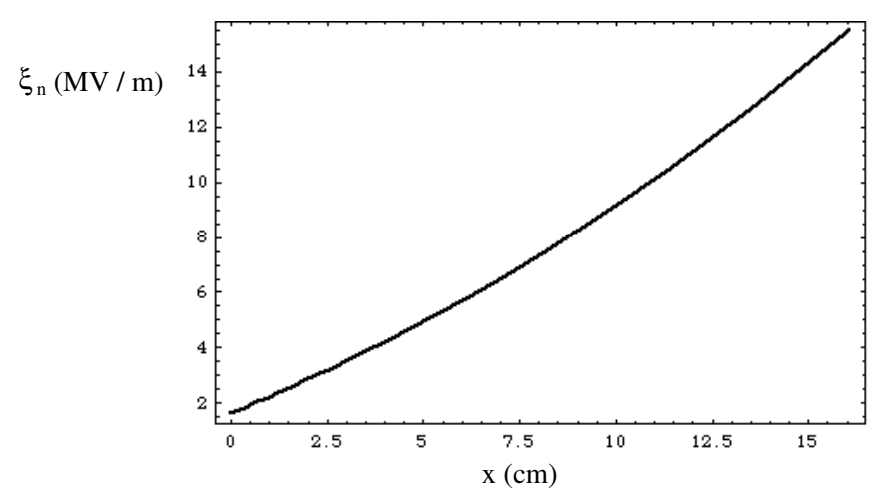

FIG. 5. (Color) Average axial field $\xi_{n}$ versus radial beam position $x$ with $\phi_{n}=60^{\circ}$. 


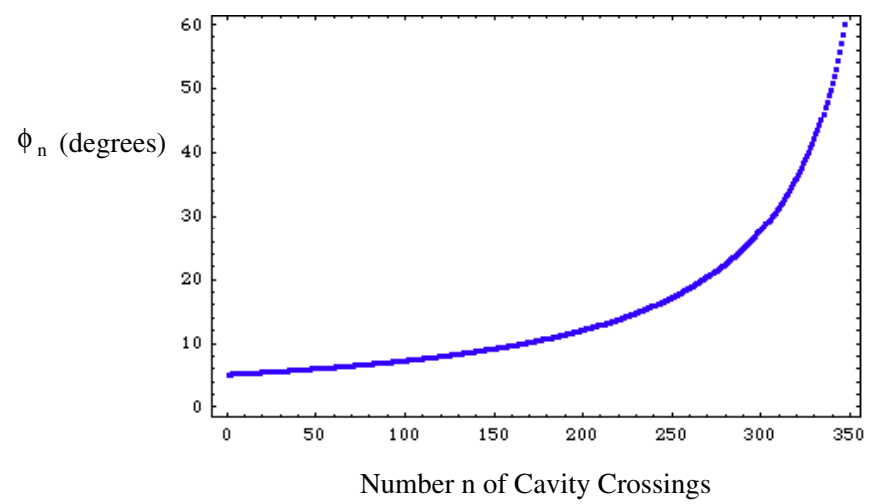

FIG. 6. (Color) rf phase $\boldsymbol{\phi}_{n}$ program versus number $n$ of cavity crossings.

method with the previous one to soften the requirements on both the change of the axial field $\xi_{n}$ and of the rf phase $\boldsymbol{\phi}_{n}$.

3. Modulation of the harmonic number jump.Inspection of Eq. (11) shows that, for the case below transition energy and $\alpha_{p n} \gamma_{n}{ }^{2} \ll 1$, the energy gain program increases with the cube of the particle total energy, for constant harmonic number jump $\Delta h$. If on the other side the latter is also programmed accordingly, for instance as $\Delta h \sim \beta_{n}{ }^{-2} \gamma_{n}{ }^{-3}$, which is a large value at injection that then decreases to unit at top energy, it is possible to reduce the variation of the axial field along the radius of the orbit. When this method is applied to the example above, we found that the required axial field is given by the plot of Fig. 7. The maximum value of $\xi_{n}$ is about unchanged, and the range of the change is considerably reduced to only a factor of two, instead of ten. As expected the acceleration rate is higher, a total of 126 revolutions against the 347 in the case of constant $\Delta h=1$. Nevertheless, because the harmonic number can decrement by only an integral amount, the shape of the axial field program is fragmented, and it is not obvious how to implement it. This method may be more useful in the case of acceleration above the transition energy when $\alpha_{p n} \gamma_{n}{ }^{2} \gg 1$. In this case the en-

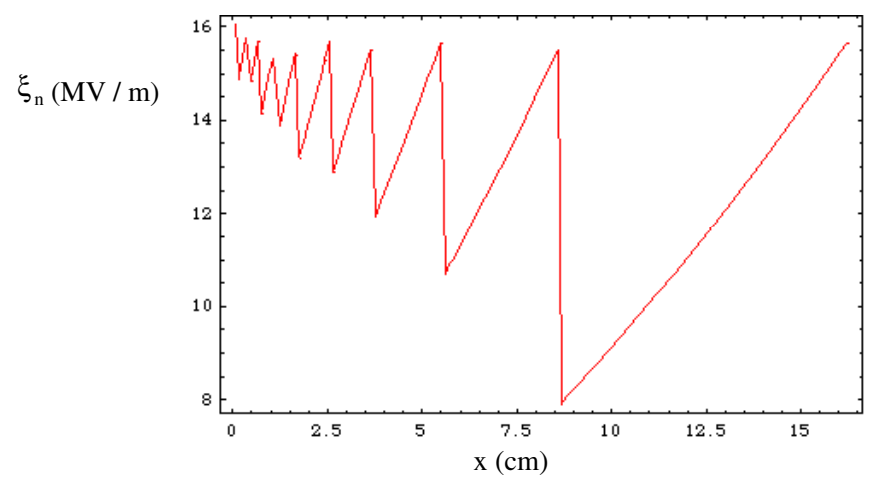

FIG. 7. (Color) Average axial field $\xi_{n}$ vs radial beam position $x$ with $\phi_{n}=60^{\circ}$ by varying the amount $\Delta h$ of the harmonic number jump during acceleration. ergy gain program varies linearly with the total particle energy. It could be flattened by allowing the amount $\Delta h$ of the harmonic number jump also to decrease, but this time only linearly, during acceleration.

4. Matching of the acceleration period with the cavity filling time. - It takes a finite amount of time to fill up with power the rf cavities which is about given by $[6,7]$

$$
T_{F}=1.4\left(Q_{0} / \omega\right) /\left(2+P_{b} / P_{w}\right)
$$

where $\omega / 2 \pi$ is the field frequency, $Q_{0}$ the unloaded figure of merit of the cavity, $P_{b}$ the beam average power, and $P_{w}$ the dissipated average power. Typically $T_{F}$ is a fraction of a millisecond and it can be made, with a proper choice of parameters, to match in magnitude the time $T_{a}$ required for acceleration over the desired energy range. In the example we have studied, the required energy gain, as seen by inspecting Fig. 4, shows about an order of magnitude in the field variation over about half a millisecond. The beam could be injected just a little after rf power is poured in the cavities. As the beam is accelerated the cavities are filled with more power until they are topped at the end of the acceleration cycle. During the filling the axial field will increase correspondingly as required.

This method sounds more feasible than it may be suggested here. Although the cavity time constant is both a tool and an impediment to tailoring the voltage profile versus time, all cavity-modulator systems are equipped with amplitude and phase loops, or full vector feedback, and so following a voltage program with high accuracy is eminently possible - even if it calls for changes which are faster than the cavity time constant, provided that they are modest and the required overpower is available.

\section{CONCLUSIONS}

We have investigated a method of low-energy hadron beam acceleration in FFAG accelerators with constant frequency rf superconducting cavities. The method requires an energy gain at each cavity adjusted to allow the beam to skip one or more rf wavelengths between cavities. The energy gain program can be obtained either by requiring a radial profile of the accelerating electric field with the proper design of the cavity, or by setting a constant accelerating field throughout the cycle together to a prescribed program of rf phase. In both approaches, transit times factors are evaluated and taken into account as the beam changes velocity over an appreciable range. It is also possible to program accordingly the harmonic number jump $\Delta h$ to maintain about constant the required axial field across the radial aperture. Also the beam could be injected and accelerated in parallel to the filling of the cavities with rf power. The analysis performed shows that the longitudinal motion occurs in rf buckets of calculable height and area. 
[1] A.G. Ruggiero, Proceedings of FFAG'05 Workshop, Osaka, Japan, 2005.

[2] A. G. Ruggiero, BNL Internal Report, C-A/AP 218, 2005.

[3] I. Gumowski, CERN/MPS/Int. RF 67-1.

[4] A.G. Ruggiero, BNL Internal Report, C-A/AP 237, 2006.
[5] A. G. Ruggiero, Nucl. Phys. B, Proc. Suppl. 155, 315 (2006); BNL Report C-A/AP/219, 2005.

[6] A. G. Ruggiero, BNL Internal Report, BNL-62312, 1995.

[7] T. Wangler, RF Linear Accelerators (John Wiley and Sons, New York, 1998), pp. 79-82. 